\title{
Hill's Equation Systems and Infinite Determinants
}

\author{
By ROBERT DenK of Regensburg
}

\begin{abstract}
In this paper a method is developed to calculate the Floquet exponents of the matrix-valued version of Hill's equation using infinite determinants. It is shown that the Floquet exponents are precisely the zeros of an infinite determinant corresponding to the differential equation. The proof of this result uses the continuity and holomorphy of the infinite determinant.
\end{abstract}

\section{Introduction}

This paper deals with ordinary differential equation systems of the form

$$
y^{\prime \prime}(x)+F(x) \cdot y(x)=0 \quad(x \in \mathbb{R}),
$$

where $F$ is a $k \times k$-matrix-valued even and periodic function. In the following we assume without loss of generality that $F$ has the period $\pi$. In the scalar case where $k=1$ this equation was first investigated by HILL [9] who also introduced the method of infinite determinants corresponding to (1). Whereas for the scalar case the theory of infinite determinants and its applications to Hill's equation has been studied by many authors, this theory was not used to deal with the matrix-valued version of Hill's equation. The aim of this paper is to show that infinite determinants can be applied to (1) for arbitrary $k$ and lead to a method to calculate the Floquet exponents of (1).

Infinite determinants were defined by POINCARÉ [19] as the limit of their finite subdeterminants. For a class of infinite matrices called normal matrices, a determinant theory was developed by von KoCH [13], [14], cf. also [11]. BóBR [1] and CoHEN [2] considered more general matrices. A determinant theory for certain linear operators in Banach spaces - starting from the Fredholm theory of integral operators - was developed by Grothendieck [8], LEŻaŃSKi [15], SIKORSKi [20], GoHBERG and KreïN [7] and others. For applications of the determinantal theory to Hill's equation, see MAGNUS [16], MENNICKEN [17] and MENNICKEN and WAGENFÜHRER [18], for instance. In connection with system (1) we will use the results of vON KoCH and some estimations from [18].

The theorem of Floquet-Lyapunov (see e.g. [23]) tells us that stability properties of the solutions of (1) can be expressed by the Floquet exponents (characteristic exponents) of 
(1), i.e., the complex numbers $v \in \mathbb{C}$ where a solution $y$ of (1) exists with $y(x+\pi)=\mathrm{e}^{i \pi v} y(x)$. In [4] it was shown that $v$ is a Floquet exponent if and only if $\cos \pi v$ is an eigenvalue of a $k \times k$-matrix that can be obtained by solving (1) with certain initial values.

The present paper gives another way to calculate the Floquet exponents: We show that these are precisely the zeros of an infinite determinant corresponding to Hill's equation (1), cf. Theorem 5.1. Moreover, this determinant is a polynomial in $\cos \pi v$ with degree $k$. This is the basis for a determinantal method to compute the Floquet exponents of (1) without integration. The proof of these results uses the holomorphy and continuity of the infinite determinant as a function of the coefficient $F$ in (1) (Section 4). After some remarks on infinite determinants in Section 2, the determinants associated to Hill's equation (1) will be defined in Section 3. This paper contains some part of the author's thesis [3].

\section{Some remarks on infinite determinants}

In this section we want to state some definitions and results of the theory of infinite determinants that will be needed later. For the following, let $\mathbf{M}(k \times k, \mathbb{C})$ be the set of all $k \times k$-matrices with complex coefficients. $\mathrm{M}(k \times k, \mathbb{C})$ will be endowed with the norm

$$
\left|\left(m_{i j}\right)_{i, j=1}^{k}\right|_{2}:=\left(\sum_{i, j}\left|m_{i j}\right|^{2}\right)^{1 / 2} .
$$

We also set

$$
\left|\left(m_{i j}\right)_{i, j=1}^{k}\right|_{\text {tr }}:=\sum_{i=1}^{k}\left|m_{i i}\right| .
$$

For a twosided infinite blockmatrix $A=\left(A_{n m}\right)_{n, m \in \mathbb{Z}}$ with $A_{n m} \in \mathrm{M}(k \times k, \mathbb{C})$ we define the finite section $A_{N}:=\left(A_{n m}\right)_{n, m=-N}^{N}$ and the determinant det $A$ by

$$
\operatorname{det} A:=\lim _{N \rightarrow \infty} \operatorname{det} A_{N}
$$

provided that the limit exists (cf. [19]).

The class $M_{2}$ is defined as the set of all matrices $A=\left(A_{n m}\right)_{n, m \in Z}$ which fulfill the condition

$$
\sum_{n \in \mathbb{Z}}\left|A_{n n}-I_{k}\right|_{\mathrm{tr}}<\infty, \quad \sum_{n, m \in \mathbb{Z}}\left|A_{n m}-\delta_{n m} I_{k}\right|_{2}^{2}<\infty .
$$

(Here $I_{k}$ denotes the unit matrix in $\mathrm{M}(k \times k, \mathbb{C})$ and $\delta_{n m}$ the Kronecker symbol.) For this class of matrices VON KOCH [13], [14] proved the existence and some properties of the determinant. The following theorem is obtained by slight modifications of the original results:

Theorem 2.1. (vON KOCH).

a) For every $A \in M_{2}$ the infinite determinant exists. $\operatorname{det} A$ is equal to zero if and only if the infinite linear system $A \cdot b=0$ has a nontrivial solution $b \in \ell^{2}\left(\mathbb{Z}, \mathbb{C}^{k}\right)$.

b) Let $A$ be a region in the complex plane and $A(\lambda)=\left(A_{n m}(\lambda)\right)_{n, m \in \mathbb{Z}}$ with holomorphic functions $A_{n m}: A \rightarrow \mathrm{M}(k \times k, \mathbb{C})$. If the series

$$
\sum_{n \in \mathbb{Z}}\left|A_{n m}(\lambda)-I_{k}\right|_{\mathrm{tr}} \quad \text { and } \sum_{n, m \in \mathbb{Z}}\left|A_{n m}(\lambda)-\delta_{n m} I_{k}\right|_{2}^{2}
$$


are bounded in every compact subset of $\Lambda$, then $\operatorname{det} A(\lambda)$ is holomorphic in $A$.

c) For all $A \in M_{2}$ and $N \in \mathbb{N}$ the finite subdeterminants det $A_{N}$ can be estimated by

$$
\left|\operatorname{det} A_{N}\right| \leq \exp \left(\sum_{n \in \mathbb{Z}}\left|A_{n n}-I_{k}\right|_{1 \mathrm{r}}+\frac{1}{2} \sum_{n, m \in \mathbb{Z}}\left|A_{n m}-\delta_{n m} I_{k}\right|_{2}^{2}\right) \text {. }
$$

By

$$
d(A, B):=\sum_{n \in \mathbb{Z}}\left|A_{n n}-B_{n m}\right|_{\mathrm{lir}}+\left(\sum_{n, m \in \mathbb{Z}}\left|A_{n m}-B_{n m}\right|_{2}^{2}\right)^{1 / 2} \quad\left(A, B \in M_{2}\right)
$$

a metric on $M_{2}$ is defined. We see from the definition that

$$
d\left(A, A_{N}\right) \rightarrow 0 \quad(N \rightarrow \infty), \quad d\left(A_{N}, B_{N}\right) \leq d(A, B)
$$

and that $M_{2}$ consists of all matrices $A$ with $d\left(A, I_{\infty}\right)<\infty$ where

$$
I_{\infty}:=\left(\delta_{n m} I_{k}\right)_{n, m \in \mathbb{Z}}
$$

is the unit matrix in $M_{2}$. (In the terms $d\left(A, A_{N}\right)$ and $d\left(A_{N}, B_{N}\right)$ we extend the finite submatrices to an infinite matrix by setting the coefficient at $(n, m)$ equal to $\delta_{n m} I_{k}$ for $|n|>N$ or $|m|>N$.) The following lemma is easily derived from Theorem 3.18 in [18]:

Lemma 2.2. Let $A \in M_{2}$ and $N_{0} \in \mathbb{N}$ with $d\left(A, A_{N_{0}}\right)<1$. Then there exists a constant $c(A)>0$ such that for all $N \geq N_{0}+1$ we get $\left|\operatorname{det} A-\operatorname{det} A_{N}\right| \leq c(A) d\left(A, A_{N}\right)$. The constant $c(A)$ is a continuous function of $A \in M_{2}$.

Using the preceding lemma and the continuity of the determinant of finite matrices we obtain the continuity of the infinite determinant by standard $\varepsilon$ - $\delta$-arguments:

Theorem 2.3. det is a continous function from $M_{2}$ to $\mathbb{C}$.

Remark 2.4. If we consider the matrix $A$ as a linear operator on $\ell^{2}$ and apply the theory of determinants in Banach spaces as described in [7], we get the continuity of the determinant as a function on the space of all nuclear operators on $\ell^{2}$ (also called trace class operators). The class $M_{2}$, however, is larger than the class of all nuclear operators, as the example of Problem 18 (p. 178) in [6] shows.

\section{Infinite determinants associated to Hill's equation}

Now we return to Hill's equation (1). We assume that

$$
\left.F\right|_{[0, \pi]} \in L^{2}([0, \pi], \mathrm{M}(k \times k, \mathbb{C})) .
$$

By a solution of equation (1) we mean an absolutely continous function $y$ with absolutely continous derivative for which (1) holds for almost every $x \in \mathbb{R}$. The solution

$$
Y_{1}: \mathbb{R} \rightarrow \mathrm{M}(k \times k, \mathbb{C})
$$


is defined by the initial values

$$
Y_{1}(0)=I_{k}, \quad Y_{1}^{\prime}(0)=0 .
$$

In [4] the following is proved:

Lemma 3.1. A complex number $v$ is a Floquet exponent of (1) if and only if $\cos \pi v$ is an eigenvalue of $Y_{1}(\pi)$.

Let $v \in \mathbb{C}$ be a Floquet exponent of (1) and $y: \mathbb{R} \rightarrow \mathbb{C}^{k}$ a corresponding Floquet solution of (1). The $\pi$-periodic function

$$
\Phi(x):=\mathrm{e}^{-i v x} y(x)
$$

and its derivative are absolutely continuous, and for almost every $x \in \mathbb{R}$ the equality

$$
\Phi^{\prime \prime}(x)=-2 i v \cdot \Phi^{\prime}(x)-\left(F(x)-v^{2} I_{k}\right) \cdot \Phi(x)
$$

holds. In particular, $\left.\Phi^{\prime \prime}\right|_{\{0, \pi]} \in L^{1}\left([0, \pi], \mathbb{C}^{k}\right)$. For any $n \in \mathbb{Z}$ the $n$-th Fourier coefficients of $\Phi$ and $F$ are defined by

$$
\begin{aligned}
& b_{n}:=\widehat{\Phi}(n)=\frac{1}{\pi} \int_{0}^{\pi} \Phi(t) \mathrm{e}^{-2 i n t} \mathrm{~d} t\left[=\frac{1}{\pi} \int_{0}^{\pi} y(t) \mathrm{e}^{-i(2 n+v) t} \mathrm{~d} t\right) \in \mathbb{C}^{k}, \\
& G_{n}:=\widehat{F}(\mathrm{n})=\frac{1}{\pi} \int_{0}^{\pi} F(t) \mathrm{e}^{-2 i n t} \mathrm{~d} t \in \mathrm{M}(k \times k, \mathbb{C}) .
\end{aligned}
$$

Due to $F(x)=F(-x)$ and $\left.F\right|_{[0, \pi]} \in L^{2}([0, \pi], \quad \mathbf{M}(k \times k, \mathbb{C}))$ we have $G_{n}=G_{-n}$ and $\left(G_{n}\right)_{n \in \mathbb{Z}} \in \ell^{2}(\mathbb{Z}, \mathrm{M}(k \times k, \mathbb{C}))$, respectively.

Now we use the fact that

$$
\left(\Phi^{\prime}\right)^{-}(n)=2 i n b_{n} \text { and }\left(\Phi^{\prime \prime}\right)^{\wedge}(n)=-4 n^{2} b_{n}
$$

([5], 2.3.4). The $n$-th Fourier coefficient of $F(x) \cdot \Phi(x)$ has the value $\sum_{j \in \mathbb{Z}} G_{n-j} b_{j}$ (cf. [5], 8.2.2). Here the infinite series is absolutely convergent, because it is the convolution of the two sequences $\left(G_{n}\right)_{n}$ and $\left(b_{n}\right)_{n}$ that are elements of $\ell^{2}(\mathbb{Z}, \mathbf{M}(k \times k, \mathbb{C}))$ and $\ell^{2}\left(\mathbb{Z}, \mathbb{C}^{k}\right)$, respectively. Comparing the $n$-th Fourier coefficient of each side of (3), we get the following lemma:

Lemma 3.2. Let $v \in \mathbb{C}$ be a Floquet exponent of (1) and $y(x)$ a corresponding Floquet solution. Then for the Fourier coefficients $b_{n} \in \mathbb{C}^{k}$, defined as above, the following equation holds:

$$
\left(G_{0}-(2 n+v)^{2} I_{k}\right) b_{n}+\sum_{j \in \mathbb{Z} \backslash\{n\}} G_{n-j} b_{j}=0 \quad(n \in \mathbb{Z})
$$

In order to apply the theory of infinite determinants, we multiply equation (4) by $\left(G_{0}-(2 n+v)^{2} I_{k}\right)^{-1}$ from the left (if the inverse exists). Let

$$
H:=\left\{z \in \mathbb{C}: \operatorname{det}\left(G_{0}-(2 n+z)^{2} I_{k}\right) \neq 0 \text { for every } n \in \mathbb{Z}\right\} .
$$


The twosided infinite blockmatrix $A(v)=\left(A_{n m}(v)\right)_{n, m \in \mathbb{Z}}\left(A_{n m}(v) \in \mathbf{M}(k \times k, \mathbb{C})\right)$ is defined by

$$
A_{n m}(v):= \begin{cases}I_{k}, & \text { if } n=m, \\ \left(G_{0}-(2 n+v)^{2} I_{k}\right)^{-1} G_{n-m}, & \text { if } n \neq m .\end{cases}
$$

From Lemma 3.2 for a Floquet exponent $v \in H$ and for the Fourier coefficients $b:=\left(b_{n}\right)_{n \in \mathbb{Z}}$ of a corresponding Floquet solution $y$ we obtain

$$
A(v) \cdot b=0 \text {. }
$$

The next theorem tells us that the theory of infinite determinants can be applied to $A(v)$ :

Theorem 3.3. For all $v \in H$ we have that $A(v) \in M_{2}$ and that the infinite determinant det $A(v)$ exists. If $v \in H$ is a Floquet exponent of (1), $\operatorname{det} A(v)=0$ holds.

Proof. The condition $\sum_{n \in \mathbf{Z}}\left|A_{n n}(v)-I_{k}\right|_{\text {tr }}<\infty$ is trivially fulfilled. We estimate the second sum in (2) by

$$
\begin{aligned}
\sum_{n, m \in \mathbb{Z}}\left|A_{n m}(v)-\delta_{n m} I_{k}\right|_{2}^{2} & =\sum_{n, m \in \mathbb{Z}}\left|\left(G_{0}-(2 n+v)^{2} I_{k}\right)^{-1} G_{n-m}\right|_{2}^{2} \\
& \leq\left(\sum_{n \in \mathbb{Z}}\left|\left(G_{0}-(2 n+v)^{2} I_{k}\right)^{-1}\right|_{2}^{2}\right) \cdot\left(\sum_{n \in \mathbb{Z}}\left|G_{n}\right|_{2}^{2}\right) .
\end{aligned}
$$

The last sum is finite because $\left(G_{n}\right)_{n} \in \ell^{2}(\mathbb{Z}, \mathbf{M}(k \times k, \mathbb{C}))$. For large enough $|n|$ we have

$$
|2 n+v|^{2} \geq 2 \cdot\left|G_{0}\right|_{2},
$$

and therefore

$$
\left|\left(G_{0}-(2 n+v)^{2} I_{k}\right)^{-1}\right|_{2} \leq \frac{2}{|2 n+v|^{2}} .
$$

Hence also the left sum is finite and we get $A(v) \in M_{2}$. From Theorem 2.1 it follows that $\operatorname{det} A(v)$ exists. If $v$ is a Floquet exponent of (1), we see from equation (5) and Theorem 2.1 that det $A(v)=0$.

\section{Lemma 3.4.}

a) $\operatorname{det} A(v)$ is a holomorphic function of $v \in H$.

b) For all $v \in H$ we have $\operatorname{det} A(v+2)=\operatorname{det} A(v)=\operatorname{det} A(-v)$.

c) $\lim _{|\operatorname{lm} v| \rightarrow \infty} \operatorname{det} A(v)=1$.

The technical proof of this lemma is based on an estimation of $d\left(A(v), I_{\infty}\right)$ which allows us to apply Theorem 2.1 and obtain the holomorphy of det $A(v)$. Part b) is a consequence of the symmetry of $A(v)$, and part c) can be proved using a continuity argument: As $A(v) \rightarrow I_{\infty}$ for $|\operatorname{Im} v| \rightarrow \infty$ in the topology of $M_{2}$, we get $\operatorname{det} A(v) \rightarrow 1$ by Theorem 2.3. For details the reader is refered to [3], Section III.2.

For any number $v_{0} \notin H$ there is a finite set of integers $\left\{n_{1}, \ldots, n_{s}\right\}$ with

$$
\operatorname{det}\left(G_{0}-\left(2 n_{j}+v_{0}\right)^{2} I_{k}\right)=0 \quad(j=1, \ldots, s) .
$$


If we multiply the $n_{j}$-th block row of $A(v)$ by $G_{0}-\left(2 n_{j}+v\right)^{2} I_{k}$ from the left $(j=1, \ldots, s)$, we obtain a modified matrix $\widetilde{A}(v)$. By the same arguments as above, we see that $\tilde{A}(v) \in M_{2}$ and that $\operatorname{det} \widetilde{A}(v)$ is holomorphic at $v_{0}$. This modification leads to the following equality for the determinants:

$$
\operatorname{det} \widetilde{A}(v)=\operatorname{det} A(v) \cdot \prod_{j=1}^{s} \operatorname{det}\left(G_{0}-\left(2 n_{j}+v\right)^{2} I_{k}\right)
$$

If $\lambda_{1}, \ldots, \lambda_{k}$ are the (not necessarily different) eigenvalues of $G_{0}$, the product on the right side of (6) has the value $\prod_{j=1}^{s} \prod_{l=1}^{k}\left(\lambda_{l}-\left(2 n_{j}+v\right)^{2}\right)$.

Lemma 3.5. The function $v \mapsto \operatorname{det}\left(\cos \pi v \cdot I_{k}-\cos \pi \sqrt{G_{0}}\right) \cdot \operatorname{det} A(v)$ is an entire function of $v \in \mathbb{C}$.

Proof. We use

$$
\begin{aligned}
\operatorname{det}\left(\cos \pi v \cdot I_{k}-\cos \pi \sqrt{G_{0}}\right) & =\prod_{j=1}^{k}\left(\cos \pi v-\cos \pi \sqrt{\lambda_{j}}\right) \\
& =\left(\frac{\pi^{2}}{2}\right)^{k} \prod_{j=1}^{k}\left\{\left(\lambda_{j}-v^{2}\right) \cdot \prod_{n \in \mathbb{Z} \backslash\{0\}} \frac{(2 n+v)^{2}-\lambda_{j}}{(2 n)^{2}}\right\} .
\end{aligned}
$$

Comparing this expression with (6), we see that for every $v \notin H$ the factor

$$
\operatorname{det}\left(\cos \pi v \cdot I_{k}-\cos \pi \sqrt{G_{0}}\right)
$$

eliminates all poles of det $A(v)$.

Remark 3.6. The term $\cos \pi \sqrt{G_{0}}$ that appears in the last lemma and similar trigonometric functions of matrices are defined by the corresponding power series. Therefore it is not necessary to assume that $\sqrt{G_{0}}$ exists (e.g. that $G_{0}$ is positive definite). The matrix $\cos \pi \sqrt{G_{0}}$ is well-defined for arbitrary $G_{0}$ (cf. [23]). The fact that the eigenvalues of $\cos \pi \sqrt{G_{0}}$ are $\cos \pi \sqrt{\lambda_{j}}$ (which was used for the above calculation of the determinants) can be seen as a special case of the spectral mapping theorem (see [10], 5.3.1, for instance).

We considered the matrix $A(v)$ in order to obtain strong convergence properties so that the theory of infinite determinants could be applied. Now we divide the $n$-th row of (4) by $(2 n)^{2}$ and obtain some infinite matrix $B(v)$. The properties of det $A(v)$ allow us to show that det $B(v)$ exists for every $v \in \mathbb{C}$; additionally, $\operatorname{det} B(v)$ is an entire function of $v \in \mathbb{C}$ :

Theorem 3.7. Let the twosided infinite blockmatrix

$$
B(v)=\left(B_{n m}(v)\right)_{n, m \in \mathbb{Z}} \quad ; \quad\left(B_{n m}(v) \in \mathbf{M}(k \times k, \mathbb{C})\right)
$$

be defined by

$$
B_{n m}(v):= \begin{cases}G_{n-m}-\delta_{n m} v^{2} I_{k}, & \text { if } n=0 \\ \frac{1}{(2 n)^{2}}\left[\delta_{n m}(2 n+v)^{2} I_{k}-G_{n-m}\right], & \text { if } n \neq 0\end{cases}
$$


Then we obtain:

a) $\operatorname{det} B(v):=\lim _{N \rightarrow \infty} \operatorname{det} B_{N}(v)$ exists for all $v \in \mathbb{C}$ and has the value

$$
\operatorname{det} B(v)=\left(\frac{2}{\pi^{2}}\right)^{k} \operatorname{det}\left(\cos \pi v I_{k}-\cos \pi \sqrt{G_{0}}\right) \cdot \operatorname{det} A(v) \quad(v \in H) .
$$

b) det $B(v)$ is an entire even function of $v$ with period 2 .

c) det $B(v)$ is a polynomial in $\cos \pi v$ with degree $k$ and leading coefficient $\left(\frac{2}{\pi^{2}}\right)^{k}$.

d) If $v \in \mathbb{C}$ is a Floquet exponent of Hill's equation (1), $\operatorname{det} B(v)=0$ holds.

Proof.

a) For every $N \in \mathbb{N}$ and $v \in H$ we can write:

$$
\operatorname{det} B_{N}(v)=\prod_{j=1}^{k}\left\{\left(\lambda_{j}-v^{2}\right) \prod_{\substack{n=-N \\ n \neq 0}}^{N} \frac{(2 n+v)^{2}-\lambda_{j}}{(2 n)^{2}}\right\} \cdot \operatorname{det} A_{N}(v) .
$$

The right side converges for $N \rightarrow \infty$ to the function

$$
\left(\frac{2}{\pi^{2}}\right)^{k} \prod_{j=1}^{k}\left(\cos \pi v-\cos \pi \sqrt{\lambda_{j}}\right) \cdot \operatorname{det} A(v) .
$$

Due to Lemma 3.5 the right side is defined for all $v \in \mathbb{C}$.

b) This follows from Lemma 3.5 and Lemma 3.4b).

c) As an entire 2-periodic and even function, det $B(v)$ can be written as a Fourier series of the form

$$
\operatorname{det} B(v)=\sum_{i=0}^{\infty} c_{l}^{\prime} \cos (l \pi v) \quad \text { with } \quad c_{l}^{\prime} \in \mathbb{C} \quad\left(l \in \mathbb{N}_{0}\right) .
$$

This series is absolutely convergent for every $v \in \mathbb{C}$. As $\cos (l \pi v)$ is a polynomial with degree $l$ in $\cos \pi v$, this Fourier series can be written as $\sum_{l=0}^{\infty} c_{l}(\cos \pi v)^{l}\left(c_{l} \in \mathbb{C}\right)$. Because of

$$
\frac{\operatorname{det} B(v)}{(\cos \pi v)^{l}}=\left(\frac{2}{\pi^{2}}\right)^{k} \frac{\prod_{j=1}^{k}\left(\cos \pi v-\cos \pi \sqrt{\lambda_{j}}\right)}{(\cos \pi v)^{l}} \cdot \operatorname{det} A(v)
$$

and $\lim _{|\operatorname{Im} v| \rightarrow \infty} \operatorname{det} A(v)=1\left(\right.$ Lemma 3.4c)), we obtain $c_{l}=0$ for $l>k$ and $c_{k}=\left(\frac{2}{\pi^{2}}\right)^{k}$.

d) In the case $v \in H$ the assertion follows immediately from $\operatorname{det} A(v)=0$ (Theorem 3.3) and part a). For $v \notin H$ consider the modified matrix $\widetilde{A}(v)$, cf. equation (6). As $\widetilde{A}(v) \cdot b=0$ holds, we have that $\operatorname{det} \widetilde{A}(v)=0$. $\operatorname{det} B(v)$ is the product of $\operatorname{det} \widetilde{A}(v)$ with some (convergent) infinite product and therefore $\operatorname{det} B(v)=0$. 


\section{Continuity and holomorphy of the determinants}

In this section we consider the determinants

$$
\operatorname{det} A(v)=\operatorname{det} A(v, F) \text { and } \operatorname{det} B(v)=\operatorname{det} B(v, F)
$$

as functions of the coefficient $F$. Here $F$ is an element of the space

$$
L_{s}^{2}:=\left\{F \in L^{2}([0, \pi], \mathbf{M}(k \times k, \mathbb{C})): F(x)=F(\pi-x) \text { almost everywhere }\right\} .
$$

This space is a closed subspace of $L^{2}([0, \pi], \mathbf{M}(k \times k, \mathbb{C}))$ and therefore a Hilbert space. In the following the fundamental solution $Y_{1}(x, F)$, the region $H(F) \subset \mathbb{C}$ and the Fourier coefficients $G_{n}(F)$ will be defined in the same way as in the previous sections.

Theorem 4.1. For $F_{0} \in L_{s}^{2}$ and $v_{0} \in H\left(F_{0}\right)$ the mapping $F \mapsto \operatorname{det} A\left(v_{0}, F\right)$ is defined in a neighbourhood of $F_{0}$ and continuous at the point $F_{0}$.

Proof. Let $F_{0} \in L_{s}^{2}$ and $v_{0} \in H\left(F_{0}\right)$. The proof is made in several steps:

(i) The mapping $F \mapsto \cos \pi \sqrt{G_{0}(F)}$ is a continous function from $L_{s}^{2}$ to $\mathrm{M}(k \times k, \mathbb{C})$. If we endow the space $\ell^{2}(\mathbb{Z}, \mathrm{M}(k \times k, \mathbb{C}))$ with the usual $\ell^{2}$-norm

$$
\left\|\left(C_{n}\right)_{n \in \mathbf{Z}}\right\|:=\sum_{n \in \mathbb{Z}}\left|C_{n}\right|_{2}^{2},
$$

then the theorem of Plancherel says that the Fourier transform is an isometry. Therefore the linear mapping $F \mapsto G_{0}(F)$ is continuous, as we see from

$$
\left|G_{0}(F)\right|_{2} \leq\left\|\left(G_{n}(F)\right)_{n \in \mathbf{Z}}\right\|_{\ell^{2}}=\|F\|_{L^{2}} .
$$

Because the function $M \mapsto \cos \pi \sqrt{M}$ is holomorphic and thus continuous, the composition $F \mapsto \cos \pi \sqrt{G_{0}(F)}$ is a continuous function (from $L_{s}^{2}$ to $\mathrm{M}(k \times k, \mathbb{C})$ ).

(ii) As $H\left(F_{0}\right)$ is an open set, there exists a circle with radius $2 r>0$ and center $v_{0}$ in the interior of $H\left(F_{0}\right)$. Using (i) and the fact that the eigenvalues of a matrix depend continuously on the coefficients, it follows that the eigenvalues of $\cos \pi \sqrt{G_{0}(F)}$ depend continuously on $F$. Thus there exists a $\delta_{1}>0$ such that for every

$$
F \in K_{\delta_{1}}\left(F_{0}\right):=\left\{F \in L_{s}^{2}:\left\|F-F_{0}\right\|_{L^{2}}<\delta_{1}\right\}
$$

and for every $z \in \mathbb{C} \backslash H(F)$ there exists a $z^{\prime} \in \mathbb{C} \backslash H\left(F_{0}\right)$ with $\left|z-z^{\prime}\right|<r$. In other words, for some $r>0$ and some $\delta_{1}>0$ the circle with center $v_{0}$ and radius $r$ lies in the interior of $H(F)$ for every $F \in K_{\delta_{1}}\left(F_{0}\right)$.

(iii) Due to (ii) $\left(G_{0}(F)-\left(v_{0}+2 n\right)^{2} I_{k}\right)^{-1}$ exists for all $F \in K_{\delta_{1}}\left(F_{0}\right)$ and for all $n \in \mathbb{Z}$. As $\frac{1}{2}\left(\sqrt{2\left|G_{0}(F)\right|_{2}}+\left|v_{0}\right|\right)$ is a continuous function of $F \in L_{s}^{2}$, there exists a $\delta_{2}>0$ such that for every $F \in K_{\delta_{2}}\left(F_{0}\right)$ we get

$$
\frac{1}{2}\left(\sqrt{2\left|G_{0}(F)\right|_{2}}+\left|v_{0}\right|\right) \leq \frac{1}{2}\left(\sqrt{2 \mid G_{0}\left(\left.F_{0}\right|_{2}\right.}+\left|v_{0}\right|\right)+1=: c .
$$


In particular,

$$
\left|2 n+v_{0}\right|^{2} \geq\left(2 N-\left|v_{0}\right|\right)^{2} \geq 2\left|G_{0}(F)\right|_{2}
$$

holds for every $N \in \mathbb{N}$ with $N \geq c$ and for every $|n| \geq N$, and we obtain the estimation

$$
\left|\left(G_{0}(F)-\left(2 n+v_{0}\right)^{2} I_{k}\right)^{-1}\right|_{2} \leq \frac{2}{\left|2 n+v_{0}\right|^{2}} \quad\left(F \in K_{\delta_{2}}\left(F_{0}\right),|n| \geq N\right) .
$$

For some $\delta_{3}>0$ the inequality

$$
\left|\left(G_{0}(F)-\left(2 n+v_{0}\right)^{2} I_{k}\right)^{-1}\right|_{2}^{2} \leq\left|\left(G_{0}\left(F_{0}\right)-\left(2 n+v_{0}\right)^{2} I_{k}\right)^{-1}\right|_{2}^{2}+1
$$

holds for all $F \in K_{\delta_{3}}\left(F_{0}\right)$ and $|n|<N$. Let $\delta:=\min \left\{\delta_{1}, \delta_{2}, \delta_{3}\right\}$. We get for all $F \in K_{\delta}\left(F_{0}\right)$ that

$$
\begin{aligned}
& \sum_{n \in \mathbb{Z}}\left|\left(G_{0}(F)-\left(2 n+v_{0}\right)^{2} I_{k}\right)^{-1}\right|_{2}^{2} \\
& \leq(2 N-1)+\sum_{|n|<N}\left|\left(G_{0}\left(F_{0}\right)-\left(2 n+v_{0}\right)^{2} I_{k}\right)^{-1}\right|_{2}^{2}+\sum_{|n| \geq N} \frac{4}{\left|2 n+v_{0}\right|^{4}}<\infty,
\end{aligned}
$$

and therefore the series converges uniformly in $K_{\delta}\left(F_{0}\right)$.

(iv) As $F \mapsto\left(G_{0}(F)-\left(2 n+v_{0}\right)^{2} I_{k}\right)^{-1}$ is continuous at the point $F_{0}$ for every $n \in \mathbb{Z}$, every term of the series

$$
\sum_{n \in \mathbb{Z}}\left|\left(G_{0}(F)-\left(2 n+v_{0}\right)^{2} I_{k}\right)^{-1}-\left(G_{0}\left(F_{0}\right)-\left(2 n+v_{0}\right)^{2} I_{k}\right)^{-1}\right|_{2}^{2}
$$

converges to 0 for $F \rightarrow F_{0}$. Using (iii) we can change the order of summation and limit, and we obtain

$$
\begin{aligned}
& \sum_{n, m \in \mathbb{Z}}\left|A_{n m}\left(v_{0}, F_{0}\right)-A_{n m}\left(v_{0}, F\right)\right|_{2}^{2} \\
& \leq 2 \sum_{n \in \mathbb{Z}}\left|\left(G_{0}\left(F_{0}\right)-\left(2 n+v_{0}\right)^{2} I_{k}\right)^{-1}\right|_{2}^{2} \cdot \sum_{n \neq 0}\left|G_{n}\left(F_{0}\right)-G_{n}(F)\right|_{2}^{2} \\
& \quad+2 \sum_{n \in \mathbb{Z}}\left|\left(G_{0}\left(F_{0}\right)-\left(2 n+v_{0}\right)^{2} I_{k}\right)^{-1}-\left(G_{0}(F)-\left(2 n+v_{0}\right)^{2} I_{k}\right)^{-1}\right|_{2}^{2} \\
& \quad \times \sum_{n \neq 0}\left|G_{n}(F)\right|_{2}^{2} \rightarrow 0
\end{aligned}
$$

for $\left\|F-F_{0}\right\|_{L^{2}} \rightarrow 0$.

(v) Using Theorem 2.3 the continuity of the function $F \mapsto \operatorname{det} A\left(v_{0}, F\right)$ follows.

Corollary 4.2. Let $F_{0} \in L_{s}^{2}$. Then for every fixed $v_{0} \in H\left(F_{0}\right)$ the mapping $F \mapsto \operatorname{det} B\left(v_{0}, F\right)$ is continuous at the point $F_{\mathrm{o}}$.

In the following we consider the set of functions $F$ whose Fourier coefficients $G_{n}(F)$ vanish for large $n$. We define

$$
\mathscr{B}_{N}:=\left\{F \in L_{s}^{2}: G_{n}(F)=0 \text { if }|n|>N\right\}
$$

and

$$
\mathscr{B}:=\bigcup_{N=0}^{\infty} \mathscr{B}_{N}
$$


(If $F \in \mathscr{B}_{N}$, equation (1) is called finite Hill's equation; in the case $F \in \mathscr{B}_{1}$, Mathieu's equation.) $\mathscr{B}_{N}$ can be identified with $\mathbb{C}^{(N+1) k^{2}}$ what allows us to define holomorphic functions from $\mathscr{B}_{N}$ to $\mathbb{C}$.

Theorem 4.3. Let $N \in \mathbb{N}$ and $v_{0} \in \mathbb{C}$ be given. $F \mapsto \operatorname{det} B\left(v_{0}, F\right)$ is a holomorphic mapping from $\mathscr{B}_{N}$ to $\mathbb{C}$.

Proof. Let $F_{0} \in \mathscr{B}_{N}$ and $v_{0} \in H\left(F_{0}\right)$. Then for some $\delta_{1}>0$ the determinant $\operatorname{det} A\left(v_{0}, F\right)$ exists for all $F \in \mathscr{B}_{N}$ with $\left\|F-F_{0}\right\|_{L^{2}}<\delta_{1}$. According to Theorem $2.1 \mathrm{c}$ ) for every $F$ with $\left\|F-F_{0}\right\|_{L^{2}}<\delta_{1}$ and for all $N \in \mathbb{N}$ we have

$$
\left|\operatorname{det} A_{N}\left(v_{0}, F\right)\right| \leq \exp \left(\frac{1}{2} \sum_{n, m \in \mathbb{Z}}\left|A_{n m}\left(v_{0}, F\right)-\delta_{n m} I_{k}\right|_{2}^{2}\right) .
$$

The continuity of $F \mapsto A\left(v_{0}, F\right)$ (cf. the proof of Theorem 4.1) gives us the existence of some $\delta<\delta_{1}$ for which the sum in (8) is bounded in $\left\{F \in \mathscr{B}_{N}:\left\|F-F_{0}\right\|_{L^{2}}<\delta\right\}$. Using the analogue of the theorem of Montel for functions of several variables (cf. [12], 2.2.5), we see that the limit of the sequence $\operatorname{det} A_{N}\left(v_{0}, F\right)$ is holomorphic at $F_{0}$.

Because of

$$
\operatorname{det} B\left(v_{0}, F\right)=\left(\frac{2}{\pi^{2}}\right)^{k} \operatorname{det}\left(\cos \pi v_{0} \cdot I_{k}-\cos \pi \sqrt{G_{0}(F)}\right) \cdot \operatorname{det} A\left(v_{0}, F\right)
$$

the function det $B\left(v_{0}, F\right)$ is a product of two holomorphic functions of $F$ and therefore itself holomorphic at $F_{0}$.

In the case $v_{0} \notin H\left(F_{0}\right)$ we consider the modified matrix $\widetilde{A}\left(v_{0}, F\right)$, and the theorem follows analogously.

\section{The main theorem}

In the last section we studied the holomorphy properties of the infinite determinant as a function of the coefficient $F$. It is well-known that the mapping $F \mapsto Y_{1}(\pi, F)$ is a continuous function from $L_{s}^{2}$ to $\mathrm{M}(k \times k, \mathbb{C})$, and from the theorem of holomorphic parameter dependence of the solution of a differential equation it follows that this mapping is a holomorphic function from $\mathscr{B}_{N}$ into $\mathrm{M}(k \times k, \mathbb{C})$ (cf. [23], II.1.7 and [23], II.1.3, respectively).

Now we want to compare the infinite determinant $\operatorname{det} B(v, F)$ and the finite determinant $\operatorname{det}\left(Y_{1}(\pi, F)-\cos \pi v I_{k}\right)$. Theorem 3.7 tells us that both determinants are polynomials in $\cos \pi v$ with degree $k$. As the next theorem will show, they are even equal up to a constant factor.

Theorem 5.1. For all $F \in L_{\mathrm{s}}^{2}$ and $v \in \mathbb{C}$ the equality

$$
\operatorname{det}\left(Y_{1}(\pi, F)-\cos \pi v \cdot I_{k}\right)=\left(-\frac{\pi^{2}}{2}\right)^{k} \operatorname{det} B(v, F)
$$

holds.

As a first step, we show that the theorem is true for $F \in \mathscr{B}$ : 
Lemma 5.2. The equation in Theorem 5.1 is valid for every $F \in \mathscr{B}$ and for every $v \in \mathbb{C}$.

Proof. For $F_{0}(x):=\left(\begin{array}{ccc}\mu_{1}^{2} & & 0 \\ 0 & \ddots & \mu_{k}^{2}\end{array}\right)$ where $\mu_{j}:=\left(\frac{1}{2}\right)^{j}(j=1, \ldots, k)$, the fundamental solution $Y_{1}\left(x, F_{0}\right)$ of equation $(1)$ is given by $\cos \left(x \sqrt{F_{0}}\right)$. Therefore

$$
\operatorname{det}\left(Y_{1}\left(\pi, F_{0}\right)-\cos \pi v I_{k}\right)=\prod_{j=1}^{k}\left(\cos \left(2^{-j} \pi\right)-\cos \pi v\right)
$$

i.e., this determinant has $k$ different zeros (as a polynomial in $\cos \pi v$ ). Due to the remarks at the beginning of this section the coefficients of det $\left(Y_{1}(\pi, F)-\cos \pi \nu I_{k}\right)$ depend continuously on $F$, and therefore there is some $\delta>0$ such that for every $F \in L_{s}^{2}$ with $\left\|F-F_{0}\right\|_{L^{2}}<\delta$ this polynomial has simple zeros. Theorem 3.7d) says that each of these zeros is also a zero of det $B(v, F)$. Because the degree of both polynomials in $k$, they possess the same zeros with the same multiplicity. Comparing the leading coefficients (see Theorem 3.7c)) we see that the theorem holds for all $F \in L_{s}^{2}$ with $\left\|F-F_{0}\right\|_{L^{2}}<\delta$.

Now let $F$ be an element of $\mathscr{B}$. Due to the definition of $\mathscr{B}$ there exists an $N \in \mathbb{N}_{0}$ with $F \in \mathscr{B}_{N}$. As we have seen above, the equality of the theorem is true for all $F^{\prime} \in \mathscr{B}_{N}$ with $\left\|F^{\prime}-F_{0}\right\|_{L^{2}}<\delta$ and thus in a neighbourhood of $F_{0} \in \mathscr{B}_{N}$. Both functions are holomorphic in $\mathscr{B}_{N}$ (Theorem 4.3), and we obtain equality in $\mathscr{B}_{N}$ (see [12], p. 27, for instance), especially for the given $F$.

Proof of Theorem 5.1. We consider some fixed $F \in L_{s}^{2}$. As $\mathscr{B}$ is a dense subset of $L_{s}^{2}$, there exists a sequence

$$
\left(F_{j}\right)_{j \in \mathbb{N}} \subset \mathscr{B} \text { with }\left\|F_{j}-F\right\|_{L^{2}} \rightarrow 0 \text {. }
$$

In the case $v \in H(F)$, from Lemma 5.2 using the continuity of the considered determinants (Corollary 4.2) we obtain

$$
\begin{aligned}
& \operatorname{det}\left(Y_{1}(\pi, F)-\cos \pi v I_{k}\right)=\lim _{j \rightarrow \infty} \operatorname{det}\left(Y_{1}\left(\pi, F_{j}\right)-\cos \pi v I_{k}\right) \\
& =\left(-\frac{\pi^{2}}{2}\right)^{k} \lim _{j \rightarrow \infty} \operatorname{det} B\left(v, F_{j}\right)=\left(-\frac{\pi^{2}}{2}\right)^{k} \operatorname{det} B(v, F) .
\end{aligned}
$$

Because both determinants are polynomials in $\cos \pi v$, we obtain this equality also in the case $v \notin H(F)$.

Corollary 5.3. A complex number $y$ is a Floquet exponent of Hill's equation (1) if and only if $\operatorname{det} B(v, F)=0$.

This corollary enables us to use the following method to calculate the Floquet exponents of Hill's equation (1): The coefficients of the polynomial det $B(v, F)$ (as a polynomial in $\cos \pi v$ ) can be computed by evaluating this determinant for $k$ suitably chosen different values of $v$ (the leading coefficient is already known). Then the Floquet exponents (precisely their cos-values) are the zeros of this polynomial. 
Finally we want to discuss briefly the case $k=1$. In this case the polynomial is of degree 1 , i.e., we get

$$
\operatorname{det} B(v, F)=\frac{2}{\pi^{2}} \cos \pi v+g(F)
$$

where $g(F)$ is a constant that does not depend on $v$. The equivalence described in the following corollary can be found in the literature (see for instance [22]) and is an almost immediate consequence of our main theorem:

Corollary 5.4. For $k=1$ the following statements are equivalent:

(i) $v$ is a Floquet exponent of Hill's equation (1).

(ii) $\sin ^{2}\left(\frac{\pi}{2} v\right)=\frac{\pi^{2}}{4} \operatorname{det} B(0, F)$.

(iii) $\cos ^{2}\left(\frac{\pi}{2} v\right)=\operatorname{det} \widetilde{B}(1, F)$ where $\widetilde{B}(1, F)=\left(\widetilde{B}_{n m}(1, F)\right)_{n, m \in \mathbb{Z}}, \widetilde{B}_{n m}(1, F) \in \mathbb{C}$, is defined by

$$
\widetilde{B}_{n m}(1, F)=\delta_{n m}-\frac{G_{n-m}}{(2 n+1)^{2}} \quad(n, m \in \mathbb{Z}) .
$$

Proof. We set $v=0$ in (9) and get $g(F)=\operatorname{det} B(0, F)-\frac{2}{\pi^{2}}$; with

$$
1-\cos \pi v=2 \sin ^{2}\left(\frac{\pi}{2} v\right)
$$

we get the equivalence of (i) and (ii). By using

$$
\widetilde{B}_{n m}(1, F)= \begin{cases}-B_{n m}(1, F) & (n=0) \\ \left(\frac{2 n}{2 n+1}\right)^{2} B_{n m}(1, F) & (\mathrm{n} \neq 0)\end{cases}
$$

$(m \in \mathbb{Z})$, we see

$$
\operatorname{det} B(1, F)=-\prod_{n=1}^{\infty}\left(1-\left(\frac{1}{2 n}\right)^{2}\right)^{2} \cdot \operatorname{det} \widetilde{B}(1, F)=-\frac{4}{\pi^{2}} \operatorname{det} \widetilde{B}(1, F),
$$

therefore

$$
g(F)=\operatorname{det} B(1, F)+\frac{2}{\pi^{2}}=\frac{2}{\pi^{2}}(1-2 \operatorname{det} \widetilde{B}(1, F))
$$

From this and Theorem 5.1 the equivalence of (i) and (iii) follows immediately.

Final remarks. As we see from the results above, the Floquet exponents of the Hill's equation (1) can be computed either by (numerical) integration or by the determinantal method. To obtain the coefficients of the infinite determinant det $B(v)$ (as a polynomial in $\cos \pi v)$ it is necessary to evaluate det $B(v)$ for $k$ different values of $v$. For numerical purposes the rate of convergence of the sequence (det $\left.B_{N}(v)\right)_{N}$ is important. In the case of finite Hill's equation, i.e., if $F \in \mathscr{B}$, it is possible to accelerate the convergence of this sequence. For 
$k=1$ and $v \in\{0,1\}$ acceleration methods can be found in papers of MENNICKEN and WAGENFÜHRER ([17], [18], [22]). Very fast methods were obtained for the scalar form of Mathicu's equation by WAGENFÜHRER [21].

In [3] acceleration methods are defined for arbitrary values of $k$ and $v$. The basis of such a method is the knowledge of the asymptotic behaviour of the sequence $\left(\operatorname{det} B_{N}(v)\right)_{N}$. If $\left(\gamma_{N}\right)_{N}$ is a sequence of complex numbers such that

$$
\operatorname{det} B_{N}(v)-\gamma_{N} \operatorname{det} B_{N-1}(v)
$$

is small, then the modified sequence $\left(b_{N}\right)_{N}$ defined by

$$
b_{N}:=\left(\prod_{n=1}^{N} \gamma_{n}\right)^{-1} \operatorname{det} B_{N}(v)
$$

will converge faster than the original sequence, and we have

$$
\operatorname{det} B(v)=\lim _{N \rightarrow \infty} b_{N} \cdot\left(\prod_{n=1}^{\infty} \gamma_{n}\right) .
$$

In [3] it is shown that (det $\left.B_{N}(v)\right)_{N}$ converges like a series with terms of order $O\left(N^{-4}\right)$. By a suitable choice of $\left(\gamma_{N}\right)_{N}$ the order $O\left(N^{-8}\right)$ can be achieved. Using this acceleration of convergence, the determinantal method leads to a fast and precise computation of the Floquet exponents. Details and numerical examples for the accelerated convergence can be found in [3].

\section{References}

[1] ST. Bobr: Eine Verallgemeinerung des von Koch'schen Satzes über die absolute Konvergenz der unendlichen Determinanten. Math. Z. 10 (1921), 1-11.

[2] L. W. CoHEN: A Note on a System of Equations with Infinite Many Unknowns. Bull. Amer. Math. Soc. 36 (2) (1930), 563-572.

[3] R. DENK: Die Determinantenmethode zur Bestimmung der charakteristischen Exponenten von Hillschen Differentialgleichungs-Systemen. Thesis, Universität Regensburg 1993.

[4] R. DenK: On the Floquet Exponents of Hill's Equation Systems. Math. Nachr. 172 (1995) 87-94

[5] R. E. EDWARDS: Fourier Series - a Modern Introduction. Holt, Rinehart and Winston Inc., New York 1967.

[6] I. Gohberg, S. Goldberg, M. A. KaAShoek: Classes of Linear Operators, Vol. I. Birkhäuser Verlag, Basel 1990.

[7] I. GOHBERG, M. G. KREÏN: Introduction to the Theory of Linear Nonselfadjoint Operators. American Mathematical Society, Providence, Rhode Island 1969.

[8] A. GRothendieck: La Théorie de Fredholm. Bull. Soc. Math. France 84 (1956), 319-384.

[9] G. W. Hill: On the Part of the Motion of the Lunar Perigee Which is a Function of the Mean Motions of the Sun and the Moon. Acta Math. 8 (1886), 1-36.

[10] E. Hille, R. Phillips: Functional Analysis and Semi-Groups. Revised Edition, American Mathematical Society, Providence 1957.

[11] E. HiLle, J. D. TAMARKIN: On the Characteristic Values of Linear Integral Equations. Acta Math. 57 (1931), 1-76.

[12] L. Hörmander: An Introduction to Complex Analysis in Several Variables. D. van Nostrand Company Inc., Princeton, New Jersey 1966.

[13] H. von Koch: Sur la Convergence des Déterminants Infinis. Rend. Circ. Mat. Palermo 28 (1909), $255-266$. 
[14] H. von Koch: Sur les Déterminants Infinis et les Équations Différentielles Linéares. Acta Math. 16 (1892), $271-295$.

[15] T. Leżański: The Fredholm Theory of Linear Equations in Banach Spaces. Studia Math. 13 (1953), 244-276.

[16] W. Wagnus: Infinite Determinants Associated with Hill's Equation. Pacific J. Math. 5, Suppl. 2 (1955), 941-951.

[17] R. MENNiCKen: On the Convergence of Infinite Hill-Type Determinants. Arch. Rational Mech. Anal. 30 (1968), $12-37$.

[18] R. MENNICKEN, E. WAGENFÜHRER: Über die Konvergenz verallgemeinerter Hillscher Determinanten. Math. Nachr. 72 (1976), $21-49$.

[19] H. Poincaré: Sur les Déterminants d'Ordre Infini. Bull. Soc. Math. France 14 (1886), 77-90.

[20] R. SiKorSKI: The Determinant Theory in Banach Spaces. Colloq. Math. 8 (1961), 141-198.

[21] E. WAGENFÜrRER: Ein Verfahren höherer Konvergenzordnung zur Berechnung des charakteristischen Exponenten der Mathieuschen Differentialgleichung. Numer. Math. 27 (1976), 53-65.

[22] E. WAGENFC̈HRER: Die Determinantenmethode zur Berechnung des charakteristischen Exponenten der endlichen Hillschen Differentialgleichung. Numer. Math. 35 (1980), 405-420.

[23] V. A. Yakubovich, V. M. STaRzhINSKII: Linear Differential Equations with Periodic Coefficients. J. Wiley \& Sons, New York 1975.

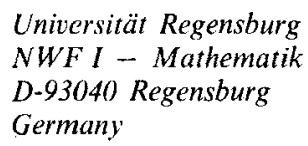

